Publ. Math. Debrecen

Manuscript

\title{
There are no proper Berwald-Einstein manifolds
}

\author{
By Shaoqiang Deng*, Dávid Csaba Kertész** and Zaili Yan*
}

\begin{abstract}
We prove that a connected Berwald-Einstein manifold is either Riemannian or Ricci-flat.
\end{abstract}

\section{Introduction}

On many occasions, S.-S. Chern raised the following question: Does every smooth manifold admit an Einstein-Finsler metric? The problem is extremely involved and has been intensely studied. However, it is still remains open, although there are several partial results.

Most of the currently available Einstein-Finsler metrics are either of Randers type or Ricci flat, see e.g., [2, 3, 6, 9, 12]. To attack the problem, it is indeed natural to consider first some special Finsler manifolds. A promising class is given by invariant Einstein-Finsler functions on homogeneous manifolds; for some results on homogeneous Einstein-Finsler functions we refer to [4]. Another important and well-understood class of Finsler manifolds is formed by Berwald manifolds. It turns out, however, that this class does not admit proper Einstein-Finsler functions. In this short note we prove the following

Theorem 1. A connected Berwald-Einstein manifold is either Riemannian or Ricci-flat.

Mathematics Subject Classification: 53C21, 53C30, 53C60.

Key words and phrases: Finsler spaces, Berwald metric, Ricci curvature.

* Supported by NSFC (11271198) and SRFDP of China.

** Supported by the Hungarian Scientific Research Fund (OTKA) Grant K-111651. 


\section{Preliminaries}

In general we follow the conventions of [1] and [11]. We denote by $M$ an $n$ dimensional connected smooth manifold. The tangent bundle and the slit tangent bundle of $M$ are $\tau: T M \rightarrow M$ and $\stackrel{\circ}{\tau}: \stackrel{\circ}{T} M \rightarrow M$, respectively. To avoid subtle technicalities, we shall frequently use local coordinates. Then $\left(u^{i}\right)$ stands for a generic local coordinate system on $M$, and $\left(x^{i}, y^{i}\right)$ is the induced local coordinate system on $T M$. (Here $x^{i}=u^{i} \circ \tau, y^{i}(v)=v\left(u^{i}\right)$.)

A Finsler function for $M$ is a positive-homogeneous function $F: T M \rightarrow$ $[0, \infty[$ such that $F$ is smooth on $\stackrel{\circ}{T} M$ and the matrix

$$
\left(g_{i j}^{F}\right):=\left(\frac{\partial^{2} \frac{1}{2} F^{2}}{\partial y^{i} \partial y^{j}}\right)
$$

is positive definite at every point of its domain. Then the pair $(M, F)$ is called a Finsler manifold.

Let $(M, F)$ be a Finsler manifold. The fundamental tensor $g_{F}$ of $(M, F)$ is the Riemannian metric on the pull-back bundle $\dot{\tau}^{*} T M$ whose components with respect to the local frame $\left(\frac{\widehat{\partial}}{\partial u^{i}}\right)_{i=1}^{n}$ induced by $\left(\frac{\partial}{\partial u^{i}}\right)_{i=1}^{n}$ are given by (1). The Finsler function $F$ induces a canonical spray on $T M$, given locally by

$$
\mathbf{G}=y^{i} \frac{\partial}{\partial x^{i}}-2 G^{i} \frac{\partial}{\partial y^{i}}
$$

where

$$
G^{i}=\frac{1}{4}\left(g^{F}\right)^{i l}\left(\frac{\partial^{2}\left(F^{2}\right)}{\partial x^{k} \partial y^{l}} y^{k}-\frac{\partial\left(F^{2}\right)}{\partial x^{l}}\right), \quad\left(\left(g^{F}\right)^{i j}\right):=\left(g_{i j}^{F}\right)^{-1} .
$$

In spite of the coordinate formulation, $\mathbf{G}$ is a globally defined $C^{1}$ vector field on $T M$, smooth on $\stackrel{\circ}{T} M$. For an intrinsic description of the canonical spray, see, e.g., [11, section 9.2.2]. The Jacobi endomorphism of $(M, F)$ (called the Riemann curvature in $[8])$ is a type $(1,1)$ tensor field on $\stackrel{\sim}{\tau}^{*} T M$, given locally by

$$
\mathbf{K}=K_{k}^{i} \frac{\widehat{\partial}}{\partial u^{i}} \otimes \widehat{d u^{k}}
$$

where $\left(\widehat{d u^{i}}\right)_{i=1}^{n}$ is the dual frame of $\left(\frac{\widehat{\partial}}{\partial u^{i}}\right)_{i=1}^{n}$ and

$$
K_{k}^{i}=2 \frac{\partial G^{i}}{\partial x^{k}}-\frac{\partial^{2} G^{i}}{\partial x^{j} \partial y^{k}} y^{j}+2 G^{j} \frac{\partial^{2} G^{i}}{\partial y^{j} \partial y^{k}}-\frac{\partial G^{i} \partial G^{j}}{\partial y^{j} \partial y^{k}} .
$$

Again, a coordinate-free definition may be found in [11]. The trace $\operatorname{Ric}_{F}=\operatorname{tr}(\mathbf{K})$ of $\mathbf{K}$ is a smooth function on $\stackrel{\circ}{T}^{M}$, called the Ricci curvature of $(M, F)$. 
Definition 2. A Finsler manifold $(M, F)$ is called a Einstein-Finsler manifold if its Ricci curvature is related to the Finsler function by

$$
\operatorname{Ric}_{F}=(\lambda \circ \tau) F^{2}=: \lambda^{\vee} F^{2},
$$

where $\lambda \in C^{\infty}(M)$.

Note that if the manifold has dimension $\geq 3$ and the Finsler function is Riemannian, then the function $\lambda$ in Definition 2 is constant by Schur's lemma [ 5 , Lemma 3]. This assertion is also true in the Randers case; see [7]. However, it is still an open problem in Finsler geometry whether the above assertion holds for a general Finsler manifold of dimension $\geq 3$, and this is the key point in our proof of Theorem 1.

Now we recall that a Finsler manifold is called a Berwald manifold if its Berwald curvature vanishes. Locally, this means that

$$
G_{j k l}^{i}=\frac{\partial G_{j k}^{i}}{\partial y^{l}}=\frac{\partial^{3} G^{i}}{\partial y^{j} \partial y^{k} \partial y^{l}}=0
$$

for all $i, j, k, l \in\{1, \ldots, n\}$, therefore the Christoffel symbols $G_{j k}^{i}$ of the Finslerian Berwald derivative 'depend only on position'. Thus there exists a family $\left(\Gamma_{j k}^{i}\right)$ of (locally defined) smooth functions such that $G_{j k}^{i}=\Gamma_{j k}^{i} \circ \tau$. The so obtained family $\left(\Gamma_{j k}^{i}\right)$ is the family of Christoffel symbols of a torsion-free covariant derivative on $M$, called the base covariant derivative of $(M, F)$. For details, we refer to [11, section 9.8].

Theorem 3. If $(M, F)$ is a Berwald manifold then there exists a Riemannian metric $g$ on $M$ whose Levi-Civita derivative is the base covariant derivative of $(M, F)$.

We note that this is just a reformulation of Z.I. Szabó's clever observation [10, Theorem 1]; see also [11, Theorem 9.8.6]. If a Riemannian metric $g$ satisfies the condition in Theorem 3 we say that $(M, F)$ and $(M, g)$ (or $F$ and $g$ ) are affine equivalent.

\section{Proof of Theorem 1}

If $\operatorname{dim} M=2$, then $(M, F)$ is automatically a locally Minkowski Finsler manifold or a Riemannian-Finsler manifold. This can be concluded from Szabó's proof of his famous structure theorem on Berwald manifolds [10, Theorem 3]. There are also direct proofs; see [1, section 10.6], or [11, section 9.9.4]. 
In order to prove Theorem 1 in the case $\operatorname{dim} M \geq 3$, we first deduce the following

Lemma 4. If a Berwald-Einstein manifold $(M, F)$ is affine equivalent to a Riemannian manifold $(M, g)$, then

$$
\lambda^{\vee} F^{2}(u)=\operatorname{Ric}_{g}(u, u) \quad \text { for all } u \in \stackrel{\circ}{T} M,
$$

where $\operatorname{Ric}_{g}$ is the Ricci tensor of $(M, g)$.

Proof. In view of (2), we have only to show that $\operatorname{Ric}_{F}(u)=\operatorname{Ric}_{g}(u, u)$. We can apply Lemma 7.15.3 and formula (8.2.4) in [11] to find that the curvature tensor $R$ of $(M, g)$ and the Jacobi endomorphism of $(M, F)$ are related by

$$
\mathbf{K}_{u}(v)=R_{p}(v, u) u ; \quad u, v \in T_{p} M, u \neq 0 .
$$

The Ricci tensor of $(M, g)$ is given by

$$
\operatorname{Ric}(X, Y)=\operatorname{tr}(Z \mapsto R(Z, X) Y) ; \quad X, Y, Z \in \mathfrak{X}(M) .
$$

Thus we have $\operatorname{Ric}_{F}(u)=\operatorname{tr}\left(v \mapsto \mathbf{K}_{u}(v)\right)=\operatorname{tr}\left(v \mapsto R_{p}(v, u) u\right)=\operatorname{Ric}_{g}(u, u)$.

Proof of Theorem 1. Suppose that $(M, F)$ is a Berwald-Einstein manifold. If the function $\lambda$ in (2) is everywhere zero then $(M, F)$ is Ricci-flat. Now assume that the set $U:=\{p \in M \mid \lambda(p) \neq 0\} \subset M$ is nonempty, and let $A$ be one of its connected components. By Theorem 3, there exists a Riemannian metric $g$ on $M$ which is affine equivalent to $F$. Then $\mathrm{Ric}_{g}$ is a symmetric bilinear form, and from Lemma 4 it follows that $(A, F)$ is Riemannian. The fundamental tensor $g^{F}$ of $(A, F)$ reduces to a Riemannian metric on $A$, denoted for simplicity by the same symbol. Then we have $F^{2}(u)=g^{F}(u, u)$ for all $u \in T A$. Since $(A, F)$ and $\left(A, g^{F}\right)$ are obviously affine equivalent, we get

$$
\lambda g^{F}(u, u)=\lambda^{\vee} F^{2}(u) \stackrel{\text { Lemma }}{=}{ }^{4} \operatorname{Ric}_{g^{F}}(u, u), \quad u \in T A .
$$

So $\left(A, g^{F}\right)$ is an Einstein manifold and $\lambda$ is constant on $A$.

We obtained that for any component $A$ of $U$, the function $\lambda \uparrow A$ is constant and $(A, F)$ is a Riemannian manifold. Since $U$ has countably many components, the image $\lambda(M) \subset \lambda(U) \cup\{0\} \subset \mathbb{R}$ of $\lambda$ must be countable. However, $M$ is connected, so $\lambda(M)$ is an interval, hence, by its countability, it consists of only a single point. Therefore $\lambda$ is constant, $U=M$, and $(M, F)$ is Riemannian.

\section{Acknowledgment}

The authors are grateful to the referee for suggesting simplifications of the original proof. 


\section{References}

[1] D. Bao, S. S. Chern And Z. Shen, An Introduction to Riemann-Finsler Geometry, Springer-Verlag, New York, 2000.

[2] D. Bao, C. Robles And Z. Shen, Zermelo navigation on Riemannian mannifold, J. Diff. Geom. 66 (2004), 377-435.

[3] X. Cheng, Z. Shen and Y. Tian, A Class of Einstein $(\alpha, \beta)$-metrics, Israel Journal of Mathematics 192 (2012), 221-249.

[4] S. Deng, Homogeneous Finsler Spaces, Springer, New York, 2012.

[5] P. Petersen, Riemannian Geometry, 2nd ed., Springer, 2006,.

[6] M. Rafie-Rad and B. Rezaei, On Einstein Matsumoto metrics, Nonlinear Anal. Real World Appl. 13, (2012), 882-886.

[7] C. Robles, Einstein metrics of Randers type, doctoral dissertation, University of British Colombia, 2003.

[8] Z. Shen, Differential Geometry of Spray and Finsler Spaces, Kluwer Academic Publishers, Dordrecht, 2001.

[9] Z. Shen, On projectively related Einstein metrics in Riemann-Finsler geometry, Math. Ann. 320 (2001), 625-647.

[10] Z. I. Szabó, Positive definite Berwald spaces, Tensor (N.S.) 35 (1981), 25-39.

[11] J. Szilasi, R. L. Lovas and D. Cs. Kertész,, Connections, Sprays and Finsler Structures, World Scientific, 2014.

[12] Y. YU AND Y. You,, On Einstein m-th root metrics, Differential Geometry and its Applications 28 (2010), 290-294.

(Zaili Yan) SCHOOL OF MATHEMATICAL SCIENCES, NANKAI UNIVERSITY, TIANJIN 300071, PEOPLE'S REPUBLIC OF CHINA

E-mail: yanzaili@mail.nankai.edu.cn

(Shaoqiang Deng) SCHOOL OF MATHEMATICAL SCIENCES AND LPMC, NANKAI UNIVERSITY, TIANJIN 300071, PEOPLE'S REPUBLIC OF CHINA

E-mail: dengsq@nankai.edu.cn

(Dávid Csaba Kertész) Institute of MATHEMAtiCs, UNIVERSity OF DEBRECEN, H-4010 DEBRECEN, P.O. BOX 12, HUNGARY

E-mail: kerteszd@science.unideb.hu 